Discrete Comput Geom 33:483-505 (2005)

DOI: $10.1007 / \mathrm{s} 00454-004-1156-9$

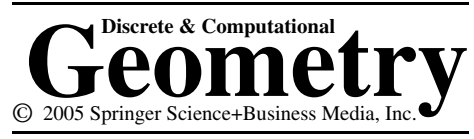

\title{
Almost Periodic Measures and Long-Range Order in Meyer Sets
}

\author{
Nicolae Strungaru \\ Department of Mathematical and Statistical Science, University of Alberta, \\ Edmonton, Alberta, Canada T6G 2G1 \\ nicolae@math.ualberta.ca \\ and \\ Institute of Mathematics, "Simion Stoilow" of the Romanian Academy, \\ Bucharest, Romania
}

\begin{abstract}
The main result of this paper is that the diffraction pattern of any Meyer set with a well-defined autocorrelation has a relatively dense set of Bragg peaks. In the second part of the paper we provide a necessary and sufficient condition for a positive pure point measure to have a continuous Fourier transform. In particular, one can get a necessary and sufficient condition for a point set to have no Bragg peaks in its diffraction.
\end{abstract}

\section{Introduction}

Within the quasicrystal community (both on the experimental and theoretical sides) it is common to equate strong long-range order with the existence of many strong Bragg peaks in the diffraction spectrum. The diffraction is described mathematically by starting with the density measure of the structure in question, say in $\mathbb{R}^{d}$, and looking at its autocorrelation, which is a positive-definite measure. The Fourier transform of this autocorrelation measure is another measure called the diffraction pattern of the structure. ${ }^{1}$ Like any measure, this one decomposes into continuous and discrete parts. We call them the continuous and discrete spectra. The set of Bragg peaks is the set of points on which the discrete spectrum is concentrated, so the question of long-range order comes down to the nature of the discrete part of this measure.

In this paper we address this question in the case when the underlying structure is a (possibly weighted) discrete set. The critical assumption that we make is that our discrete sets are Meyer sets ( $M$ is a Meyer set if $M$ is relatively dense and $M-M$ is uniformly discrete). The Meyer sets were introduced by Meyer in [15], and fully described by Moody in [16].

\footnotetext{
${ }^{1}$ For the basis on diffraction and quasicrystals one can consult [7], [9], [13], [14] and [20].
} 
In the particular case of lattice subsets, the following result was proved by Baake:

Proposition 1.1 [3]. Let $L$ be a lattice in $\mathbb{R}^{d}$ and let $S \subset$ L. Let $L^{*}$ be the dual lattice of $L$. Then the diffraction pattern of $S$ is $L^{*}$-invariant. In particular, each of the continuous and discrete spectrums is either zero or supported on a relatively dense set.

We prove (Proposition 3.12) that, under the Meyer set assumption, there is always a relatively dense set of Bragg peaks. Also the continuous spectrum is either zero or supported on a relatively dense set.

Another equivalent definition for a Meyer set is that the set is relatively dense and is also a subset of a model set [16]. The fact that the Meyer sets must have non-trivial Bragg peaks come from the relative denseness. If one looks at the diffraction pattern for an arbitrary subset of a model set, one gets the same result as in the lattice case: each of the continuous and discrete spectrums is either zero or supported on a relatively dense set. This shows a connection between model sets and lattices, a connection which seems natural from cut and project schemes, but is difficult to prove in general.

The well-known Riemann-Lebesgue lemma tells us that the Fourier transform of any absolutely continuous measure must vanish at infinity. This gives a necessary condition for the autocorrelation of a point set to have an absolutely continuous diffraction spectrum. In the case of translation bounded measures, a necessary and sufficient condition for a measure to have a continuous Fourier transform is given in [12]. The condition (namely, null-weak almost periodicity) is mentioned below, but generally is not easy to check. In the particular case of positive translation bounded measures we provide an equivalent asymptotic condition. In particular, we get a simple necessary and sufficient condition on the asymptotic behavior of the autocorrelation coefficients for the diffraction to be a continuous measure (i.e. no Bragg peaks). Thus one can consider this as a clean characterization of long-range order.

Although physical applications are concerned with $\mathbb{R}^{d}$ (when $d=2,3$ ), much of the theory that we develop is valid for (compactly generated) $\sigma$-compact, locally compact Abelian groups, and we use this setting as long as we can.

The key results derive from the theory of translation bounded and almost periodic measures, so for much of the paper we do not need to specialize to the setting of some underlying structure and its autocorrelation. However, when we do come to the question of diffraction from point sets $M$ and the existence of a discrete component in the diffraction pattern, we depend critically on the Meyer property. The Meyer condition is quite strong. Obviously it would be good to relax this assumption, but so far it has been an important component in most work on diffraction in mathematical quasicrystals, and it might represent some natural barrier beyond which long-range order becomes considerably more complex.

As we said before, we are interested in the continuous and discrete components of the diffraction spectrum of a discrete point set $M$. Mathematically we have to study the Fourier transform of the autocorrelation measure. In Section 2 we collect the definitions and results that we need from the theory of measures and their Fourier transforms.

We conclude this Introduction by briefly recalling some standard definitions from the theory of aperiodic point sets. 
Throughout the entire paper we consider $G$ to be a $\sigma$-compact, locally compact Abelian group. We denote its Haar measure by $\theta$.

Definition 1.2. For two compact sets $A, K \subset G$ we define the $K$-boundary of $A$ by

$$
\partial^{K} A=\left((K+A) \backslash A^{\circ}\right) \cup((-K+\overline{G \backslash A}) \cap A) .
$$

Definition 1.3. A sequence $\mathcal{A}=\left\{A_{n}\right\}_{n}$ of compact sets $A_{n} \subset G$ is called a van Hove sequence $^{2}$ if for all compact sets $K \subset G$ we have

$$
\lim _{n \rightarrow \infty} \frac{\theta\left(\partial^{K}\left(A_{n}\right)\right)}{\theta\left(A_{n}\right)}=0
$$

For a measure $\mu$ on $G$ we denote $\tilde{\mu}$ for the measure defined by

$$
\tilde{\mu}(E)=\overline{\mu(-E)},
$$

for all measurable sets $E \subset G$.

We say that a set $S \subset G$ is locally finite if $S \cap K$ is finite for all compact sets $K \subset G$.

Given a locally finite set $S \subset G$ we define $\delta_{S}$ by

$$
\delta_{S}=\sum_{x \in S} \delta_{x}
$$

where $\delta_{x}$ is the normalized point measure at $x$.

Definition 1.4. Given a locally finite point set $S$ and a van Hove sequence $\left\{A_{n}\right\}_{n}$, we say that $S$ has a well-defined autocorrelation with respect to $\left\{A_{n}\right\}_{n}$, if the sequence

$$
\eta_{n}=\frac{\delta_{S \cap A_{n}} * \tilde{\delta}_{S \cap A_{n}}}{\theta\left(A_{n}\right)}
$$

converges in the vague topology to a measure $\eta$. We call this measure the autocorrelation of $S$.

We use the notation $\mathcal{K}(G)=\{f: G \rightarrow \mathbb{C} \mid f$ is continuous and has compact support\}.

Let $\mathcal{K}_{2}(G)$ be the subspace of $\mathcal{K}(G)$ spanned by $\{f * g \mid f, g \in \mathcal{K}(G)\}$.

Definition 1.5. A measure $\mu$ on a locally compact Abelian group $G$ is called Fourier transformable if and only if there exists a measure $\widehat{\mu}$ on the dual group $\widehat{G}$, called the Fourier transform of $\mu$, such that

$$
\langle\widehat{\mu}, g\rangle=\langle\mu, \widehat{g}\rangle,
$$

for all $g \in \mathcal{K}_{2}(G)$, where $\widehat{g}$ denotes the Fourier transform of the function $g$.

\footnotetext{
${ }^{2}$ See the Appendix for more about van Hove sequences.
} 
The basic properties of Fourier transformable measures can be found in Chapter 1 of [6] or Chapters 10-11 of [12].

The autocorrelation of a point set is a positive definite measure. Thus it is Fourier transformable [6].

\section{Almost Periodic Measures}

Definition 2.1. Let $S$ be a locally finite set. Suppose that its autocorrelation $\eta$ exists with respect to some van Hove sequence. Then we call $\hat{\eta}$ the diffraction measure (or pattern) of $S$.

Thus, if $\eta$ is the autocorrelation and $\widehat{\eta}$ its Fourier transform, we are interested in $(\widehat{\eta})_{p p}$ and $(\widehat{\eta})_{c}$. In the autocorrelation case the theory of Gil de Lamadrid and Argabright [12] can be used, so one gets a unique decomposition $\eta=\eta_{S}+\eta_{0}$, into the strong and null weakly almost periodic components, such that

$$
\begin{aligned}
(\widehat{\eta})_{p p} & =\left(\widehat{\eta_{S}}\right), \\
(\widehat{\eta})_{c} & =\left(\widehat{\eta_{0}}\right) .
\end{aligned}
$$

We explain below the decomposition $\eta=\eta_{S}+\eta_{0}$.

Restrict for a moment to $G=\mathbb{R}^{d}$. We can write

$$
(\widehat{\eta})_{p p}=\sum_{x \in A} c_{x} \delta_{x}
$$

where $A$ is a countable set. Let $\eta_{S}$ be the part of the autocorrelation which is mapped by Fourier transform into $(\widehat{\eta})_{p p}$. Then, using the inverse Fourier transform, we should get

$$
\eta_{S} \sim \sum_{x \in A} c_{x} e^{2 \pi\langle x, \cdot\rangle}
$$

The problem here is to determine in what sense is the second sum convergent. Anyhow, this is similar to the Bohr approximation of almost periodic functions, so the answer should be similar. In [12] the authors proved that $\eta_{S}$ is almost periodic in a sense that we describe below, and they studied both the measures $\eta_{S}$ and $\eta_{0}=\eta-\eta_{S}$.

Definition 2.2. In the spirit of [12] we use the following notations:

$$
\begin{aligned}
C(G) & =\{f: G \rightarrow \mathbb{C} \mid f \text { continuous }\}, \\
C_{\mathrm{B}}(G) & =\{f \in C(G) \mid f \text { bounded }\}, \\
C_{\mathrm{U}}(G) & =\left\{f \in C_{\mathrm{B}}(G) \mid f \text { uniformly continuous }\right\}, \\
C_{0}(G) & =\left\{f \in C_{\mathrm{U}}(G) \mid f \text { vanishing at } \infty\right\} .
\end{aligned}
$$

All are Banach spaces with respect to $\|\cdot\|_{\infty}$. 
For each $x \in G$ we define $\tau_{x}: C_{\mathrm{U}}(G) \rightarrow C_{\mathrm{U}}(G)$ by

$$
\left(\tau_{x} f\right)(y)=f(-x+y) .
$$

Definition 2.3. We define the weak topology on $C_{\mathrm{U}}(G)$ as the topology defined by its dual space. We refer to the Banach topology as the strong topology.

Definition 2.4. A measure $\mu$ on $G$ is called translation bounded if for every compact set $K \subset G$ there exists a constant $C$ so that

$$
|\mu|(x+K) \leq C, \quad \forall x \in G
$$

We denote the set of translation bounded measures on $G$ by $\mathcal{M}^{\infty}(G)$.

Definition 2.5. The map $\mu \rightarrow\{\mu * f\}_{\{f \in \mathcal{K}(G)\}}$ is an embedding of $\mathcal{M}^{\infty}(G)$ in $\left[C_{\mathrm{U}}(G)\right]^{\mathcal{K}(G)}$. Giving $\left[C_{\mathrm{U}}(G)\right]^{\mathcal{K}(G)}$ the usual product topology, the induced topology on $\mathcal{M}^{\infty}(G)$ is called the product topology. We also refer to this topology as the strong topology. The weak topology is defined by the dual space of $\mathcal{M}^{\infty}(G)$.

Remark 2.6. The product topology defines a structure of locally convex topological vector space on $\mathcal{M}^{\infty}(G)$. A fundamental system of semi-norms is given by $\left\{\|\cdot\|_{f}\right\}_{f \in \mathcal{K}(G)}$, where

$$
\|\mu\|_{f}:=\|\mu * f\|_{\infty}
$$

Definition 2.7. Let $\mu$ be a translation bounded measure on $G$. Let $D_{\mu}=\left\{\delta_{x} * \mu\right\}_{x \in G}$ and $C_{\mu}=$ the closed ${ }^{3}$ convex hull of $D_{\mu}$. We say that $\mu$ is amenable (see [11] $]^{4}$ or page 52 of [12]) if and only if $C_{\mu}$ contains exactly one scalar multiple $\mu_{0}$ of the Haar measure $\theta$. In this case we write

$$
\mu_{0}=M(\mu) \theta
$$

and call $M(\mu)$ the mean of $\mu$.

We say that $f \in C_{\mathrm{U}}(G)$ is amenable if and only if the measure $f d \theta$ is amenable. If this happens we define $M(f)=M(f d \theta)$.

\section{Remark 2.8.}

(i) $\mathrm{f}$ is amenable if and only if $C_{f}$, the closed convex hull of $\left\{\delta_{x} * f\right\}_{x \in G}$ contains exactly one constant function. In this case the constant is $M(f)$.

\footnotetext{
${ }^{3}$ A theorem by Mazuro and Bourgin [8] says that in a locally convex topological vector space a convex set is closed if and only if it is weakly closed. Thus the closure in this definition is the same in both strong and weak topologies.

${ }^{4}$ Eberlein uses a more general setting and the concept of ergodicity. In the particular case of $\mathcal{M}^{\infty}(G)$, Definition 3.1 of an ergodic element becomes the one we use for amenability.
} 
(ii) This definition is difficult to use. In [11] it is proved that for any amenable function the averaged integral is well defined if one computes the average over some particular sequences. We prove later in this paper that the average integral can be computed over van Hove sequences.

It is easy to see that, if it is well defined, the average integral is constant on $C_{f}$. Thus, if a function is amenable, the mean is the average integral.

Definition 2.9. $f \in C_{\mathrm{U}}(G)$ is called strongly almost periodic if $C_{f}$ is compact in the strong topology. $f$ is called weakly almost periodic if $C_{f}$ is compact in the weak topology. $f$ is called null weakly almost periodic if $|f|$ is amenable, weakly almost periodic, and $M(|f|)=0$.

We denote by $\operatorname{SAP}(G), \operatorname{WAP}(G)$ and $\mathrm{WAP}_{0}(G)$ the spaces of strongly, weakly and null weakly almost periodic functions on $G$, respectively.

Remark 2.10. In a Banach space a closed set is compact if and only if its closed convex hull is compact [10], [19]. Thus the previous definition is equivalent to the usual one for almost periodic functions: that the closure of $D_{f}$ is compact. We prefer to use the one with $C_{f}$ because we use the same set for both weak and strong topology.

These definitions extend to translation bounded measures.

Definition 2.11. A measure $\mu \in \mathcal{M}^{\infty}(G)$ is called strongly almost periodic if $C_{\mu}$ is compact in the product topology and $\mu$ is called weakly almost periodic if $C_{\mu}$ is compact in the weak topology. We denote by $\mathcal{S} \mathcal{A P}(G)$ and $\mathcal{W} \mathcal{A P}(G)$ the spaces of strongly and weakly almost periodic measures on $G$, respectively. A translation bounded measure $\mu$ over a locally compact Abelian group $G$ is called null weakly almost periodic if and only if for each $g \in \mathcal{K}(G), g * \mu$ is a null weakly almost periodic function. The corresponding space of measures is denoted by $\mathcal{W} \mathcal{A} \mathcal{P}_{0}(G)$.

Remark 2.12 [12, Corollary 5.4 and Corollary 5.5]. For these properties we can talk about a duality between measures and functions. More precisely, if $P$ is the property of being strongly, weakly, null weakly almost periodic or amenable, then the following are true:

(i) $f \in C_{\mathrm{U}}(G)$ has property $P$ if and only if $f d \theta \in \mathcal{M}^{\infty}(G)$ has property $P$.

(ii) $\mu \in \mathcal{M}^{\infty}(G)$ has property $P$ if and only if $f * \mu$ has property $P$ for every $f \in \mathcal{K}(G)$.

Remark 2.13. Using Remark 2.12 we can see that any null weakly almost periodic measure is in fact a weakly almost periodic one.

We make use of the following results:

Proposition 2.14 [6], [12]. Let $\mu$ be a positive definite measure. Then

(i) $\mu \in \mathcal{M}^{\infty}(G)$,

(ii) $\mu$ is Fourier transformable. 
Proposition 2.15 [2]. Let $\mu$ be a transformable measure on $G$, with $\widehat{\mu}$ translation bounded and Fourier transformable. Then $\mu \in \mathcal{W} \mathcal{A} \mathcal{P}(G)$.

Proof. We apply Theorem 11.1 of [12] to the inverse Fourier transform of $\mu$.

Proposition 2.16 [12, Theorems 7.2 and 8.1]. Let $\mu \in \mathcal{W} \mathcal{A P}(G)$. Then $\mu$ can be written uniquely in the form

$$
\mu=\mu_{S}+\mu_{0}
$$

with $\mu_{S} \in \mathcal{S} \mathcal{A P}(G), \mu_{0} \in \mathcal{W} \mathcal{A} \mathcal{P}_{0}(G)$.

Proposition 2.17 [12, Theorem 11.2]. Let $\mu$ be a transformable measure and let $\widehat{\mu}$ be translation bounded and Fourier transformable. Then

$$
\begin{aligned}
(\widehat{\mu})_{p p} & =\left(\widehat{\mu_{S}}\right), \\
(\widehat{\mu})_{c} & =\left(\widehat{\mu_{0}}\right) .
\end{aligned}
$$

Corollary 2.18. Let $\mu$ be a transformable measure and let $\widehat{\mu}$ be translation bounded. Then $\mu$ is a pure point measure if and only if $\widehat{\mu} \in \mathcal{S A P}(G)$ and $\mu$ is a continuous measure if and only if $\widehat{\mu} \in \mathcal{W} \mathcal{A} \mathcal{P}_{0}(G)$.

Remark 2.19. Suppose that $\mu \in \mathcal{M}^{\infty}\left(\mathbb{R}^{d}\right)$ is Fourier transformable. Then $\widehat{\mu}$ is Fourier transformable and

$$
\widehat{\widehat{\mu}}=\tilde{\mu} .
$$

To see this, one may use the following argument suggested by Baake. $\widehat{\mu}$ is well defined in the tempered distribution sense and $\widehat{\hat{\mu}}=\tilde{\mu}$, when viewed as tempered distributions. Thus $\langle\tilde{\mu}, g\rangle=\langle\widehat{\mu}, \widehat{g}\rangle, \forall g \in \mathcal{S}\left(\mathbb{R}^{d}\right)$.

Any function in $\mathcal{K}\left(\mathbb{R}^{d}\right)$ can be approximated by a function in the Schwartz class $S\left(\mathbb{R}^{d}\right)$, so one gets

$$
\langle\tilde{\mu}, g\rangle=\langle\widehat{\mu}, \widehat{g}\rangle, \quad \forall g \in \mathcal{K}_{2}\left(\mathbb{R}^{d}\right)
$$

\section{The Diffraction of Meyer Sets}

The main result of the paper is that the set of Bragg peaks of a Meyer set in $\mathbb{R}^{d}$ is relatively dense.

We use the fact that regular model sets are pure point diffractive (see [21] or [5]) and that they are uniformly distributed to prove that in this case $\eta_{S}$ and $\eta_{0}$ are pure point measures. Thus the pure point and continuous diffraction spectra must be strong almost periodic measures. Also, the relative denseness of a Meyer set will show that $\eta_{S} \neq 0$. For any almost periodic function the set of $\varepsilon$-almost periods is relatively dense. Thus if the function is non-identically zero, its support must be a relatively dense set. We will translate this remark to measures, and thus get the desired result. 
For this section we work with $G=\mathbb{R}^{d}$. We need this when we use the fact that any Meyer set is a subset of a model set. We work with respect to a fixed, but arbitrary van Hove sequence $\mathcal{A}=\left\{A_{n}\right\}_{n \in \mathbb{N}}$.

Definition 3.1. Let $A$ be a Delone set in $\mathbb{R}^{d}$ and suppose that its autocorrelation $\eta$ exists. Let $B=\left\{x \in \mathbb{R}^{d} \mid \widehat{\eta}(\{x\}) \neq 0\right\}$. $B$ is called the set of Bragg peaks of $A$.

Lemma 3.2. Let $M$ be a Meyer set in $\mathbb{R}^{d}$. Then $M$ is subset of a regular model set.

Proof. An $M$ Meyer set implies that $M$ is a subset of a model set [16]. Let $H$ be the internal space of this model set and let $W$ be its window. Let $U$ be an open set in $H$ such that $\bar{U}$ is compact and $\theta_{H}(\partial U)=0 . \bar{W}$ is compact implies $\bar{W} \subset \bigcup_{i=1}^{n}\left(t_{i}+U\right)$ for some $t_{1}, \ldots, t_{n} \in H$. Let $V:=\bigcup_{i=1}^{n}\left(t_{i}+U\right)$, then $V$ is open, has compact closure and $\theta_{H}(\partial V)=0$.

Let

$$
\Lambda=\Lambda(V),
$$

in the notation of [16]. Then $M \subset \Lambda$ and $\Lambda$ is a regular model set.

Proposition 3.3. Let $M$ be a subset of a regular model set $\Lambda \subset \mathbb{R}^{d}$ and suppose that its autocorrelation $\eta$ exists with respect to $\mathcal{A}$. Let

$$
\eta=\eta_{S}+\eta_{0}
$$

be the decomposition into the strongly and null-weakly almost periodic parts. Then $\eta_{S}, \eta_{0}$ are pure point measures.

Proof. Let $\eta_{\Lambda}$ be the autocorrelation of $\Lambda$. We know [17] that the autocorrelation of a regular model set exists with respect to any van Hove sequence and is independent of its choice.

We know $\Lambda$ is a regular model set hence $\Lambda$ is pure point diffractive [5]. Thus $\eta_{\Lambda}=$ $\left(\eta_{\Lambda}\right)_{S}$.

Since the strongly almost periodic component of a positive measure is positive [12, Proposition 7.2] and $\eta \geq 0$ we get $\eta_{S} \geq 0$. Now,

$$
\begin{gathered}
M \subset \Lambda \Rightarrow \eta \leq \eta_{\Lambda} \Rightarrow \eta_{\Lambda}-\eta \geq 0 \Rightarrow\left(\eta_{\Lambda}-\eta\right)_{S} \geq 0, \\
\left(\eta_{\Lambda}-\eta\right)_{S} \geq 0, \eta_{\Lambda}=\left(\eta_{\Lambda}\right)_{S} \Rightarrow \eta_{S} \leq \eta_{\Lambda} .
\end{gathered}
$$

So we get

$$
0 \leq \eta_{S} \leq \eta_{\Lambda}, \quad \text { hence } \operatorname{supp}\left(\eta_{\mathrm{S}}\right) \subset \operatorname{supp}\left(\eta_{\Lambda}\right) \subset \Lambda-\Lambda .
$$

In particular $\eta_{S}$ is a pure point measure,

$$
\eta_{0}=\eta-\eta_{S} \Rightarrow \operatorname{supp}\left(\eta_{0}\right) \subset \operatorname{supp}\left(\eta_{\mathrm{S}}\right) \cup \operatorname{supp}(\eta) \subset(\Lambda-\Lambda) \cup(\mathrm{M}-\mathrm{M})=\Lambda-\Lambda .
$$


Proposition 3.4. Let $0 \neq f \in C_{\mathrm{U}}(G)$ be a strongly almost periodic function and let $A=\operatorname{supp}(f)$. Then $G$ can be covered by finitely many translates of $A$.

Proof. $\quad f \neq 0 \Rightarrow f(x) \neq 0$ for some $x \in G$. Since $\tau_{-x} f$ is strongly almost periodic and $\operatorname{supp}\left(\tau_{x} f\right)=x+A$, without loss of generality we may assume that $f(0) \neq 0$. Let $0<\varepsilon<|f(0)|$.

For $g \in C_{\mathrm{U}}(G)$ we define $B_{\varepsilon}(g)=\left\{h \in C_{\mathrm{U}}(G) \mid\|g-h\|_{\infty}<\varepsilon\right\}$. $B_{\varepsilon}(g)$ is an open set. $D_{f}=\left\{\tau_{x} f\right\}_{x \in G}$ is a subset of $C_{f}$ which implies that $D_{f}$ has compact closure. It is easy to see that

$$
\overline{D_{f}} \subset \bigcup_{x \in G} B_{\varepsilon}\left(\tau_{x} f\right) .
$$

Since $\overline{D_{f}}$ is compact, there exists $x_{1}, \ldots, x_{n} \in G$ such that

$$
\overline{D_{f}} \subset \bigcup_{i=1}^{n} B_{\varepsilon}\left(\tau_{x_{i}} f\right) .
$$

So for any fixed $x \in G$ there is an $i$ such that $\tau_{x} f \in B_{\varepsilon}\left(\tau_{x_{i}} f\right)$. Thus, we get

$$
\left\|\tau_{x} f-\tau_{x_{i}} f\right\|_{\infty}<\varepsilon \quad \text { and }\left|\tau_{x} f\left(x_{i}\right)-\tau_{x_{i}} f\left(x_{i}\right)\right|<\varepsilon<|f(0)| .
$$

Consequently,

$$
\begin{aligned}
\left|f\left(-x+x_{i}\right)-f(0)\right|<f(0), & \text { hence } f\left(-x+x_{i}\right) \neq 0, \\
& \text { hence }-x+x_{i} \in A \text {, so } x \in x_{i}-A .
\end{aligned}
$$

Since $x \in G$ was arbitrary, we get $G \subset \bigcup_{i=1}^{n}\left(x_{i}-A\right)$, thus $G=-G \subset \bigcup_{i=1}^{n}\left(-x_{i}+A\right)$.

Proposition 3.5. The support of any non-trivial strongly almost periodic measure on $G$ is relatively dense.

Proof. Let $\mu$ be a non-trivial strongly almost periodic measure on $G$. Let $A=\operatorname{supp}(\mu)$. Let $f \in \mathcal{K}(G)$ be a non-negative function such that $f \neq 0$, and let $K=\operatorname{supp}(f)$. If $0 \neq f * \mu(x)=\int_{G} f(x-y) d \mu(y)=\int_{A} f(x-y) d \mu(y)$ then there exists $y \in A$ such that $f(x-y) \neq 0$, so $x-y \in K$, hence $x \in K+A$. Thus we get that $f * \mu \equiv 0$ on $G \backslash(K+A)$. Since $K+A$ is closed we get

$$
\operatorname{supp}(f * \mu) \subset A+K .
$$

Since $\mu$ is strongly almost periodic we get that $f * \mu$ is a strongly almost periodic function by Remark 2.12. Applying the previous proposition we get that there exist $x_{1}, \ldots, x_{n} \in G$ so that

$$
G \subset \bigcup_{i=1}^{n}\left(x_{i}+\operatorname{supp}(f * \mu)\right) \subset \bigcup_{i=1}^{n}\left(x_{i}+A+K\right)=\bigcup_{i=1}^{n}\left(x_{i}+K\right)+A,
$$

proving that $A$ is relatively dense. 
Remark 3.6. Let $S$ be a Delone set and suppose that its autocorrelation $\eta$ exists with respect to $\mathcal{A}$. Since $\eta$ is positive and positive definite, we know that $\eta_{S}$ is positive and positive definite. Thus each of $\eta$ and $\eta_{S}$ are Fourier transformable and $\widehat{\eta}$ and $\widehat{\eta_{S}}$ are also Fourier transformable. Taking the difference we get that $\eta_{0}$ and $\widehat{\eta}_{0}$ are also Fourier transformable.

Thus we can apply Corollary 2.18 for $\eta, \eta_{S}$ and $\eta_{0}$.

Proposition 3.7. Let $S$ be subset of a regular model set, and suppose that its autocorrelation $\eta$ exists with respect to $\mathcal{A}$. Then each of $\operatorname{supp}(\widehat{\eta})_{p p}$ and $\operatorname{supp}(\widehat{\eta})_{c}$ is either relatively dense or empty.

Proof. We know from Proposition 3.3 that $\eta_{S}$ and $\eta_{0}$ are pure point measures. Thus, from Corollary 2.18 , we get that $(\widehat{\eta})_{p p}$ and $(\widehat{\eta})_{c}$ are strongly almost periodic measures. The result now follows from Proposition 3.5.

Definition 3.8. Let $S$ be a Delone set in $\mathbb{R}^{d}$, and suppose that its autocorrelation $\eta$ exists with respect to $\mathcal{A}$. We say that $S$ is pure point diffractive if $\widehat{\eta}$ is a pure point measure.

Proposition 3.9. Let $S$ be a set in $\mathbb{R}^{d}$ which has finite local complexity (i.e. $S-S$ is locally finite), and suppose that $S$ is pure point diffractive. Then $S$ has a relatively dense set of Bragg peaks.

Proof. Let $\eta$ be the autocorrelation of $S$ and let $\widehat{\eta}$ be its Fourier transform. Since $\eta$ is pure point we get that $\widehat{\eta}$ is strongly almost periodic. We know that $\widehat{\eta}$ is also pure point. Then the set of Bragg peaks is dense in its support. Applying Proposition 3.5 we get that the set of Bragg peaks is relatively dense.

Lemma 3.10. Let $A$ be a relatively dense set in $\mathbb{R}^{d}$ and let $B$ be another subset of $\mathbb{R}^{d}$ such that $A \subset B$ and $B$ has finite local complexity. Then there exists a finite set $F$ such that

$$
B \subset A+F \text {. }
$$

Proof. Let $R>0$ be such that $\mathbb{R}^{d}=A+B_{R}(0)$, and define $F=(B-A) \cap B_{R}(0)$. $F$ is finite since $F \subset(B-B) \cap B_{R}(0)$. Let $y \in B$. Since $\mathbb{R}^{d}=A+B_{R}(0)$ we get $y=x+z$ with $x \in A$ and $z \in B_{R}(0)$. However, $z=y-x \in B-A$, hence $z \in F$. This proves that $B \subset A+F$.

Lemma 3.11. Let $M$ be a Meyer set and suppose that its autocorrelation $\eta$ exists with respect to $\mathcal{A}$. Then there exists a regular model set $\Lambda$ and a finite set $F$ such that

$$
\eta_{\Lambda} \leq \sum_{x, y \in F} \tau_{(x-y)} \eta
$$

In particular, $\eta_{S} \neq 0$. 
Proof. Let $\Lambda$ be a regular model set so that $M \subset \Lambda$. Then, from Lemma 3.10, we get that there exists a finite set $F$ with $\Lambda \subset F+M$. Let $t \in M-M$. Then

$$
\begin{aligned}
\frac{\#\left((\Lambda \cap(t+\Lambda)) \cap A_{n}\right)}{\lambda\left(A_{n}\right)} & \leq \frac{\#\left(((M+F) \cap(t+M+F)) \cap A_{n}\right)}{\lambda\left(A_{n}\right)} \\
& =\frac{\#\left(\bigcup_{x, y \in F}((M+y) \cap(t+M+x)) \cap A_{n}\right)}{\lambda\left(A_{n}\right)} \\
& \leq \frac{\sum_{x, y \in F} \#\left(((M+y) \cap(t+M+x)) \cap A_{n}\right)}{\lambda\left(A_{n}\right)} \\
& =\sum_{x, y \in F} \frac{\#\left(((M+y) \cap(t+M+x)) \cap A_{n}\right)}{\lambda\left(A_{n}\right)},
\end{aligned}
$$

where $\lambda$ is Lebesgue measure in $\mathbb{R}^{d}$.

Now letting $n \rightarrow \infty$ and using the fact that the autocorrelation of $\Lambda$ exists with respect to any van Hove sequence we get

$$
\eta_{\Lambda} \leq \sum_{x, y \in F} \tau_{(x-y)} \eta
$$

For the last claim we see that

$$
\eta_{\Lambda}=\left(\eta_{\Lambda}\right)_{S} \leq\left(\sum_{x, y \in F} \tau_{(x-y)} \eta\right)_{S}=\sum_{x, y \in F}\left(\tau_{(x-y)} \eta\right)_{S}=\sum_{x, y \in F} \tau_{(x-y)} \eta_{S} .
$$

Since $\Lambda$ is relatively dense we get that $\eta_{\Lambda}(\{0\}) \neq 0$, hence $\eta_{S} \neq 0$.

Proposition 3.12. Let $M$ be a Meyer set and suppose that its autocorrelation $\eta$ exists with respect to $\mathcal{A}$. Then the set of Bragg peaks is relatively dense. Moreover, if $M$ is not pure point diffractive, it has a relatively dense support for the continuous spectrum as well.

Proof. Since $M$ is a Meyer set we get that $M$ is a subset of a regular model set. Now the proof is a consequence of Proposition 3.7 and Lemma 3.11.

Remark 3.13. In [22] we show that there is a connection between the set of Bragg peaks for a Meyer set and the $\varepsilon$-dual sets.

If one reads the proofs carefully, one sees that the only property of Meyer sets we use to get Proposition 3.12 is that any Meyer set is a subset of a pure point diffractive set with finite local complexity.

Thus one gets:

Proposition. Let $\Lambda$ be a pure point diffractive Delone set with finite local complexity, and let $A \subseteq \Lambda$. Then each of the discrete and continuous diffraction spectra is either 0 or supported on a relatively dense set. Moreover, if $A$ is relatively dense, then the set of Bragg peaks is relatively dense. 
Proposition 3.14. Let $M$ be a Meyer set. Let $D$ be a Delone set such that $M \subset D$ and suppose that the autocorrelation $\eta_{D}$ exists with respect to $\mathcal{A}$. Then $D$ has an infinite set of Bragg peaks.

Proof. Suppose by way of contradiction that $D$ has only finitely many Bragg peaks.

We take any regular model set $\Lambda$ such that $M \subset \Lambda$. Then there exists $F$ finite for which $\Lambda \subset M+F \subset D+F$. Just as in Lemma 3.11 we get

$$
\eta_{\Lambda} \leq \sum_{x, y \in F} \tau_{(x-y)} \eta_{D}
$$

Looking at the strongly almost periodic parts we get

$$
\eta_{\Lambda} \leq \sum_{x, y \in F} \tau_{(x-y)}\left(\eta_{D}\right)_{S}
$$

However, $\eta_{\Lambda}$ is a non-trivial pure point measure, hence $\left(\left(\eta_{D}\right)_{S}\right)_{p p} \neq 0$.

Since $D$ has only finitely many Bragg peaks, $\left(\widehat{\eta_{D}}\right)_{p p}=\sum_{z \in B} c_{z} \delta_{z}$ for some finite set $B$. Thus

$$
\widehat{\left(\eta_{D}\right)_{S}}=\widehat{\widehat{\left(\eta_{D}\right)_{S}}}=\widehat{\left(\widehat{\eta_{D}}\right)_{p p}}=\sum_{z \in B} c_{z} e^{-2 \pi i\langle z, \cdot}
$$

is a continuous measure. However, this contradicts the fact that $\left(\left(\eta_{D}\right)_{S}\right)_{p p} \neq 0$.

Remark 3.15. We used the intermediate regular model set $\Lambda$ to prove that $\left(\left(\eta_{D}\right)_{S}\right)_{p p} \neq$ 0 because we do not know whether the autocorrelation of $M$ exists with respect to $\mathcal{A}$. One could also use the fact that for any point set $C$ and any van Hove sequence there exists a subsequence with respect to which the autocorrelation of $C$ exists [4].

Definition 3.16. Let $D$ be a locally finite subset of $G$. We say that $D$ has $\mathcal{A}$-zero density if

$$
\lim _{n \rightarrow \infty} \sup \frac{\#\left(D \cap A_{n}\right)}{\theta\left(A_{n}\right)}=0 .
$$

We say that two sets $B$ and $C$ are $\mathcal{A}$-statistically the same if $B \triangle C$ has $\mathcal{A}$-zero density. We say that $B$ is an $\mathcal{A}$-statistical Meyer set if there exists a Meyer set $M$ such that $B$ and $M$ are $\mathcal{A}$-statistically the same.

\section{Remark 3.17.}

(i) If two sets are $\mathcal{A}$-statistically the same, and the autocorrelation of one can be computed with respect to $\mathcal{A}$, then the autocorrelation of the other can be computed with respect to $\mathcal{A}$ and the autocorrelations are equal.

(ii) For a given set $B$ we can find a set $C$ which is $\mathcal{A}$-statistically the same as $B$ and contains a Meyer subset if and only if $B$ contains an $\mathcal{A}$-statistical Meyer subset.

(iii) For more properties of the statistical equality, see [18]. 
Corollary 3.18. Let $S$ be a Delone set whose autocorrelation exists with respect to the van Hove sequence $\mathcal{A}$. If $S$ contains an $\mathcal{A}$-statistical Meyer set, then $S$ has an infinite set of Bragg peaks.

Proof. From the previous remark we know that we can find a set $B$ which contains a Meyer set and has the same autocorrelation as $S$. Applying Proposition 3.14 we are done.

\section{The Diffraction of Weighted Combs with Meyer Set Support}

Throughout this section we work with a real weighted comb:

$$
\omega=\sum_{x \in M} \omega(\{x\}) \delta_{x},
$$

where $M$ is a Meyer set. We require that $\omega$ is a translation bounded measure. Also, for the entire section we consider a fixed, but arbitrary van Hove sequence $\mathcal{A}=\left\{A_{n}\right\}_{n \in \mathbb{N}}$.

We say that the autocorrelation of $\omega$ exists with respect to $\mathcal{A}$ if

$$
\eta(z)=\lim _{n \rightarrow \infty} \frac{1}{\lambda\left(A_{n}\right)} \sum_{\substack{x, y \in S \cap A_{n} \\ x-y=z}} \omega(\{x\}) \overline{\omega(\{y\})}
$$

exists for all $z \in M-M$. In this case we define

$$
\eta=\sum_{z \in M-M} \eta(z) \delta_{z}
$$

and we call it the autocorrelation of $\omega$.

For the properties of the autocorrelation see [5]. There it is also shown that in this case the definition is equivalent to the previous one.

We further assume that the autocorrelation exists with respect to $\mathcal{A}$, and that the autocorrelation $\eta_{M}$ of $M$ exists with respect to $\mathcal{A}$. This is possible since, if we pick an arbitrary van Hove sequence, the autocorrelation of $\omega$ exists for a subsequence [4] and we can repeat the argument for $M$.

We assume for the entire section that $\widehat{\eta}$ and $(\widehat{\eta})_{p p}$ are also Fourier transformable.

Proposition 4.1. Let $\hat{\eta}$ be a Fourier transform of the autocorrelation and let $B$ be the smallest set where the pure point component of $\hat{\eta}$ is concentrated..$^{5}$ Let also $\left\{D_{k}\right\}_{k \in \mathbb{N}} \subset \mathbb{R}^{d}$ be an arbitrary van Hove sequence. Then the following are equivalent:

(i) $B$ is non-empty;

(ii) $\hat{\eta}$ is not continuous;

(iii) $\lim _{k \rightarrow \infty}\left(|\eta|\left(D_{k}\right) / \lambda\left(D_{k}\right)\right) \neq 0$ (i.e. either the limit does not exist or it exists and is not zero);

(iv) $B$ is relatively dense;

(v) $B$ is an infinite set.

${ }^{5} B$ is the intersection of all the concentration sets for $(\hat{\eta})_{p p}$. Its closure is $\operatorname{supp}(\hat{\eta})_{p p}$. 
Proof. (iv) $\Rightarrow$ (v) $\Rightarrow$ (i) $\Leftrightarrow$ (ii) are trivial. (ii) $\Leftrightarrow$ (iii) follows from Proposition 5.7, proved in the next section. (ii) $\Rightarrow$ (iv) Let $C$ be such that $|\omega(\{x\})|<C$ for all $x \in M$. Then $-C \delta_{M} \leq \omega \leq C \delta_{M}$. It is easy to see that

$$
-C^{2} \eta_{M} \leq \eta \leq C^{2} \eta_{M} .
$$

Since $\left(\eta_{M}\right)_{S}$ is a pure point measure we get that $\eta_{S}$ is a pure point measure.

Now, since $\hat{\eta}$ is not continuous, we obtain $\hat{\eta}_{p p} \neq 0$. However, we know that $\hat{\eta}_{p p}=\widehat{\eta}_{S}$, so $\hat{\eta}_{p p}$ has a pure point Fourier transform, hence is strongly almost periodic. Applying Proposition 3.5 we get that $B$ is relatively dense.

Remark 4.2. A similar argument shows that if there exists $r>0$ for which $\omega(\{x\})>r$ for all $x \in M$, then the support of the pure point part of the diffraction pattern of $\omega$ is relatively dense. Moreover, in this case, $\eta$ is positive and positive definite, thus $\widehat{\eta}$ and $(\widehat{\eta})_{p p}$ are Fourier transformable.

\section{Translation Bounded Measures with Continuous Fourier Transform}

In the case when $\mu$ is positive we provide a necessary and sufficient condition for $\widehat{\mu}$ to be continuous. The idea behind the proof is that whenever $f$ and $\mu$ are positive, we can ignore the absolute value in the definition of null-weak almost periodicity.

We want the mean of $f * \mu$ to be zero. The key to the result is that $\left.(f * \mu)\right|_{A}$ is different from $f *\left(\left.\mu\right|_{A}\right)$ only on the $K$-boundary of $A$, for some compact $K$. The integral of the second function over $G$ is just $\mu(A) \int_{G} f$. Thus, in this case, the average integral of $f * \mu$ is just the average of $\mu$ multiplied with $\int_{G} f$.

Proposition 5.1 [12, Corollary 11.1]. Let $\mu$ be a translation bounded transformable measure on $G$ and suppose that $\widehat{\mu}$ is transformable and translation bounded. Then $\widehat{\mu}$ is a continuous measure if and only if $\mu \in \mathcal{W} \mathcal{A} \mathcal{P}_{0}(G)$.

Definition 5.2. For $g \in \mathcal{K}(G)$ let

$$
\theta(g):=\int_{G} g(x) d \theta(x) .
$$

Proposition 5.3. Let $\mu$ and $g \in \mathcal{K}(G)$ be positive. Let $K=-\operatorname{supp}(g) \cup \operatorname{supp}(g)$ and let $A, B \subset G$ be two sets such that $A+K \subset B$. Then

(i) $\left(\left.\mu\right|_{A}\right) * g \leq\left.(\mu * g)\right|_{B}$,

(ii) $\left.(\mu * g)\right|_{A} \leq\left(\left.\mu\right|_{B}\right) * g$.

Proof. (i) Let $y \in G$. $\left(\left.\mu\right|_{A}\right) * g(y)=\int_{G} g(y-x) d\left(\left.\mu\right|_{A}\right)(x)=\int_{A} g(y-x) d \mu(x)$. If $y \notin A+K$ then $\left(\left.\mu\right|_{A}\right) * g(y)=0$ and $\left.(\mu * g)\right|_{B} \geq 0$. Thus (i) holds. If $y \in A+K$ then $y \in B$, so

$$
\left.(\mu * g)\right|_{B}(y)=\mu * g(y)=\int_{G} g(y-x) d \mu(x) \geq \int_{A} g(y-x) d \mu(x),
$$

and again (i) holds. 
(ii) Let $y \in G$. If $y \notin A$ then $\left.(\mu * g)\right|_{A}(y)=0 \leq\left(\left.\mu\right|_{B}\right) * g$. If $y \in A$ then $y+K \subset B$. Then

$$
\begin{aligned}
\left(\left.\mu\right|_{B}\right) * g(y) & =\int_{G} g(y-x) d\left(\left.\mu\right|_{B}\right)(x)=\int_{y-\operatorname{supp}(g)} g(y-x) d\left(\left.\mu\right|_{B}\right)(x) \\
& =\int_{y+K} g(y-x) d \mu(x),
\end{aligned}
$$

the last equality following the fact that $y+K \subset B$. Since $g(y-x) \neq 0$ only if $x \in y+K$ we get

$$
\left(\left.\mu\right|_{B}\right) * g(y)=\int_{G} g(y-x) d \mu(x) \geq\left.(\mu * g)\right|_{A}(y) .
$$

Proposition 5.4. If $\mu$ is positive, translation bounded and transformable and $g \in \mathcal{K}(G)$ is positive, then $g * \mu$ is amenable and

$$
M(g * \mu)=\theta(g) \lim _{n \rightarrow \infty} \frac{\mu\left(A_{n}\right)}{\theta\left(A_{n}\right)}
$$

for any sequence $\left\{A_{n}\right\}_{n}$ which has the M-property. ${ }^{6}$ In particular, this is true if $\left\{A_{n}\right\}_{n}$ is a van Hove sequence.

Proof. Since $\mu$ is transformable we know that $\mu$ is a weakly almost periodic measure [12, Theorem 11.1]. Hence $\mu * g$ is an amenable function [12, Corollary 5.4] and

$$
M(g * \mu)=\lim _{n \rightarrow \infty} \frac{\int_{A_{n}^{\prime}} g * \mu(x) d \theta}{\theta\left(A_{n}^{\prime}\right)},
$$

for any sequence $\left\{A_{n}^{\prime}\right\}_{n}$ which has the Følner property. In particular, we obtain that the limit exists.

Let $K=-\operatorname{supp}(g) \cup \operatorname{supp}(g)$. From Proposition 5.3 we have

$$
\int_{A_{n}^{K-}} g * \mu(x) d \theta \leq \int_{G} g *\left(\left.\mu\right|_{A_{n}}\right)(x) d \theta \leq \int_{A_{n}^{K+}} g * \mu(x) d \theta .
$$

Since everything is positive, by the Tonelli Theorem we can change the order of integration. Thus we get

$$
\begin{aligned}
\int_{G} g *\left(\left.\mu\right|_{A_{n}}\right)(x) d \theta & =\int_{G}\left(\int_{G} g(x-y) d\left(\left.\mu\right|_{A_{n}}\right)(y)\right) d \theta \\
& =\int_{G} \int_{G} g(x-y) d \theta d\left(\left.\mu\right|_{A_{n}}\right)(y) \\
& =\int_{G} \theta(g) d\left(\left.\mu\right|_{A_{n}}\right)(y)=\theta(g) \mu\left(A_{n}\right) .
\end{aligned}
$$

\footnotetext{
${ }^{6}$ See the Appendix for the definition.
} 
So we get

$$
\int_{A_{n}^{K-}} g * \mu(x) d \theta \leq \theta(g) \mu\left(A_{n}\right) \leq \int_{A_{n}^{K+}} g * \mu(x) d \theta .
$$

Dividing by $\theta\left(A_{n}\right)$ we get

$$
\frac{\int_{A_{n}^{K-}} g * \mu(x) d \theta}{\theta\left(A_{n}^{K-}\right)} \frac{\theta\left(A_{n}^{K-}\right)}{\theta\left(A_{n}\right)} \leq \theta(g) \frac{\mu\left(A_{n}\right)}{\theta\left(A_{n}\right)} \leq \frac{\int_{A_{n}^{K+}} g * \mu(x) d \theta}{\theta\left(A_{n}^{K-}\right)} \frac{\theta\left(A_{n}^{K-}\right)}{\theta\left(A_{n}\right)} .
$$

Using (2) and the definition of $M$-sequences we get that first and last term converge to $M(g * \mu)$, so

$$
M(g * \mu)=\theta(g) \lim _{n \rightarrow \infty} \frac{\mu\left(A_{n}\right)}{\theta\left(A_{n}\right)} .
$$

Corollary 5.5. If $\mu$ is a positive transformable measure on $G$ and $\left\{A_{n}\right\}_{n}$ has the $\mathbf{M}$ property then

$$
M(\mu)=\lim _{n \rightarrow \infty} \frac{\mu\left(A_{n}\right)}{\theta\left(A_{n}\right)}
$$

Proof. In Corollary 5.4 of [12] it is proved that if $g \in \mathcal{K}(G)$ is positive with $\theta(g)=1$, then

$$
M(\mu)=M(\mu * g) .
$$

Hence the result follows from Proposition 5.4.

Corollary 5.6. If $\mu$ is a positive, translation bounded and transformable measure, and $\left\{A_{n}\right\}$ has the M-property (in particular if $\left\{A_{n}\right\}$ is a van Hove sequence), then the following are equivalent:

(i) $\mu \in \mathcal{W} \mathcal{A} \mathcal{P}_{0}(G)$,

(ii) $\mu$ has continuous Fourier transform,

(iii) $\lim _{n \rightarrow \infty}\left(\mu\left(A_{n}\right) / \theta\left(A_{n}\right)\right)=0$,

(iv) $M(\mu)=0$.

Proof. We already stated that (i) $\Leftrightarrow$ (ii) and (iii) $\Leftrightarrow$ (iv).

(i) $\Rightarrow$ (iii) Let $g \in \mathcal{K}(G)$ be a positive function, not identical to zero. Then $\theta(g)>0$. By Proposition 5.4,

$$
0=M(|g * \mu|)=M(g * \mu)=\theta(g) \lim _{n \rightarrow \infty} \frac{\mu\left(A_{n}\right)}{\theta\left(A_{n}\right)} .
$$

Dividing by $\theta(g) \neq 0$ we obtain (iii).

(iii) $\Rightarrow$ (i) Let $g \in \mathcal{K}(G)$ be arbitrary. It is easy to see that

$$
M(|g * \mu|) \leq M(|g| * \mu)
$$

Applying Proposition 5.4 to $|g| \in \mathcal{K}(G)$ we obtain $M(|g| * \mu)=0$. Since $M(|g * \mu|) \geq 0$ we are done. 
We use $|\mu|$ for the variation measure of $\mu$.

Proposition 5.7. Let $\mu$ be a transformable, translation bounded measure and let $\widehat{\mu}$ be translation bounded. Let $\left\{A_{n}\right\}$ have the $\mathbf{M}$-property (in particular, let $\left\{A_{n}\right\}$ be any van Hove sequence). Consider the following statements:

(i) $\mu \in \mathcal{W} \mathcal{A} \mathcal{P}_{0}(G)$,

(ii) $\mu$ has continuous Fourier transform,

(iii) $|\mu|$ has continuous Fourier transform,

(iv) $|\mu| \in \mathcal{W}_{\mathcal{A}} \mathcal{P}_{0}(G)$,

(v) $\lim _{n \rightarrow \infty}\left(|\mu|\left(A_{n}\right) / \theta\left(A_{n}\right)\right)=0$,

(vi) $M(|\mu|)=0$.

Then (iii) $\Leftrightarrow$ (iv) $\Leftrightarrow$ (v) $\Leftrightarrow$ (vi) $\Rightarrow$ (i) $\Leftrightarrow$ (ii) always. Moreover, if $\operatorname{supp}(\mu)$ is uniformly discrete, all statements are equivalent.

Proof. The equivalence (iii) $\Leftrightarrow$ (iv) $\Leftrightarrow$ (v) $\Leftrightarrow$ (vi) is obvious from Corollary 5.6, and (i) $\Leftrightarrow$ (ii) follows from Proposition 5.1.

(iii) $\Rightarrow$ (i) Let $g \in \mathcal{K}(G)$ be arbitrary. It is easy to see that $|g * \mu| \leq|g| *|\mu|$. Thus $M(|g * \mu|) \leq M(|g| *|\mu|)$. Using (iii) we obtain $M(|g * \mu|)=0$. Thus $\mu \in \mathcal{W} \mathcal{A} \mathcal{P}_{0}(G)$.

We prove now that, under the assumption $\operatorname{supp}(\mu)$ is uniformly discrete, (i) and (vi) are equivalent. Let $S:=\operatorname{supp}(\mu)$ and let $U$ be open such that $(S-S) \cap U=\{0\}$. Let $V$ be an open neighborhood of 0 such that $V-V \subset U$. Since $S=\operatorname{supp}(\mu)$ is uniformly discrete then $\mu=\sum_{s \in S} c_{s} \delta_{s}$ for some $c_{s} \in \mathbb{R}$, and it is easy to see that $|\mu|=\sum_{s \in S}\left|c_{s}\right| \delta_{s}$.

Let $g \in \mathcal{K}(G)$ be arbitrary so that $K:=\operatorname{supp}(g) \subset V$. We prove that $|g * \mu|=|g| *|\mu|$ :

$$
\begin{aligned}
g * \mu(x) & =\int_{G} g(x-y) d \mu(y) \\
& =\sum_{y \in S} g(x-y) \mu(\{y\}) .
\end{aligned}
$$

If $y \notin x-K$ then $x-y \notin K$, hence $g(x-y)=0$. Thus

$$
g * \mu(x)=\sum_{y \in S \cap(x+K)} g(x-y) \mu(\{y\}) .
$$

By the choice of $V$ we have $\#(S \cap(x-K)) \leq 1$, whence

$$
\left|\sum_{y \in S \cap(x-K)} g(x-y) \mu(\{y\})\right|=\sum_{y \in S \cap(x-K)}|g(x-y) \mu(\{y\})|
$$

and

$$
\begin{aligned}
|g * \mu(x)| & =\sum_{y \in(S \cap(x-K))}|g(x-y) \mu(y)| \\
& =\sum_{y \in(S \cap(x-K))}|g(x-y)||\mu(y)|=|g| *|\mu|(x) .
\end{aligned}
$$

Since $0=M(|g * \mu|)=M(|g| *|\mu|)$, applying Corollary 5.6 we are done. 
A natural question now is can we replace the condition of uniform discreteness by a weaker one? The natural condition to think of is weak uniform discreteness. The following is an example of a null weakly almost periodic measure which does not satisfy the third condition of Proposition 5.7:

Example 5.8. Let $\mu=\sum_{n \in \mathbb{Z} \backslash\{0\}}\left(\delta_{n}-\delta_{n+1 / n}\right) \in \mathcal{M}^{\infty}(\mathbb{R})$. Let $A_{n}=[-n, n]$. Then $\mu \in \mathcal{W} \mathcal{A} \mathcal{P}_{0}(\mathbb{R})$, but

$$
\lim _{n \rightarrow \infty} \frac{|\mu|\left(A_{n}\right)}{\lambda\left(A_{n}\right)}=\lim _{n \rightarrow \infty} \frac{\sum_{s \in \operatorname{supp}(\mu) \cap A_{n}}|\mu(s)|}{\lambda\left(A_{n}\right)}=2 .
$$

Proof. The only thing which is not trivial is that $\mu \in \mathcal{W} \mathcal{A} \mathcal{P}_{0}(\mathbb{R})$, so we concentrate on this.

First we prove that for all $g \in K(\mathbb{R}), g * \mu$ is a function vanishing at $\infty$. Let $k$ be an integer such that $\operatorname{supp}(g) \subset[-k, k]$. Let $\varepsilon>0$ be arbitrary. $g \in K(\mathbb{R})$ implies that $g$ is uniformly continuous. Thus there exists a $\delta>0$ such that $|x-y|<\delta$ implies $|g(x)-g(y)|<\varepsilon /(2 k+2)$.

Let $n_{0}>0$ be such that $1 / n_{0}<\delta$. Let $|n|>n_{0}$. Let $x \in \mathbb{R}$. Then

$$
\left|\left(\delta_{n}-\delta_{n+1 / n}\right) * g(x)\right|<\varepsilon /(2 k+2) .
$$

Moreover, supp $\left(\left(\delta_{n}-\delta_{n+1 / n}\right) * g\right) \subset[-n-k-1 / n, k-n] \subset[-n-k-1, k-n]$.

Let $|y|>n_{0}+k+1$. Then $\left(\delta_{n}-\delta_{n+1 / n}\right) * g(y) \neq 0$ only if $y \in[-n-k-1, k-n]$, thus only if $n \in[-y-k-1, k-y]$. However, this implies that there are at most $2 k+2$ terms of the type $\left(\delta_{n}-\delta_{n+1 / n}\right) * g(y)$ we have to consider in $\mu * g(y)$. Also $n \in[-y-k-1, k-y]$ implies $|n| \geq|y|-k-1>n_{0}$. Thus, for any $n \in[-y-k-1, k-y]$, we have $\left|\left(\delta_{n}-\delta_{n+1 / n}\right) * g(y)\right|<\varepsilon /(2 k+2)$. Thus, for all $y$ with $|y|>n_{0}+k+1$, we have $|\mu * g(y)|<\varepsilon$. Now since $\mu * g$ is vanishing at $\infty$ we get $\mu * g$ is null weakly almost periodic [11]. Since this is true for any $g \in \mathcal{K}(G)$, by Remark 2.12 we get $\mu \in \mathcal{W} \mathcal{A} \mathcal{P}_{0}(\mathbb{R})$.

Remark 5.9. For any pure point measure $\mu$, the following implications are true:

$$
M(|\mu|)=0 \Rightarrow \mu \in \mathcal{W} \mathcal{A} \mathcal{P}_{0}(G) \Rightarrow M(\mu)=0 .
$$

We saw that if $\mu$ is positive all three are equivalent. We saw that the first two are equivalent under the assumption of uniform discreteness, but not equivalent for weakly uniform discreteness. We construct an example of pure point measure $\mu$ with uniformly discrete support so that $M(\mu)=0$, but $\mu \notin \mathcal{W} \mathcal{A} \mathcal{P}_{0}(G)$ :

Let

$$
\mu=\sum_{n \in \mathbb{Z}}(-1)^{n} \delta_{n}
$$

It is obvious that $\mu$ is transformable (because it is periodic) and $M(\mu)=0$.

Now, since $\operatorname{supp}(\mu)=\mathbb{Z}$ we know

$$
\mu \in \mathcal{W} \mathcal{A} \mathcal{P}_{0}(G) \Leftrightarrow M(|\mu|)=0
$$

However $M(|\mu|)=1$. In fact, $\mu \in \mathcal{S} \mathcal{A} \mathcal{P}(G)$, with

$$
\widehat{\mu}=\delta_{(2 \mathbb{Z}+1) / 2} \text {. }
$$




\section{Acknowledgments}

I thank my supervisor Dr. Robert Moody and Dr. Michael Baake for their support and guidance during this paper. I also thank them for many good discussions and useful suggestions.

\section{Appendix. Averaging Sequences}

The setting for all averaging processes in this paper is that of van Hove sequences. In Section 5 we needed various results, which require particular specialized properties of a sequence to prove them. In this section we discuss these properties, particularly the property $\mathbf{M}$, and show that all van Hove sequences satisfy it. One can find these results in [23] or Appendix 3 of [24]. We provide the proofs here because the discussion in [24] is in a more general context and, also, some of the propositions there are mentioned without proofs.

Definition A.1. Let $\left\{A_{n}\right\}_{n}$ be a sequence of measurable subsets of $G$. We say that the sequence has the Følner property if

$$
\lim _{n} \frac{\theta\left(A_{n} \Delta\left(x+A_{n}\right)\right)}{\theta\left(A_{n}\right)}=0 \quad \text { for all } \quad x \in G
$$

Definition A.2. Let $\left\{A_{n}\right\}_{n \in \mathbb{N}}$ be a sequence of measurable sets in $G$. We say that this sequence has the property $\mathbf{M}$ if:

(i) $\left\{A_{n}\right\}_{n}$ has the Følner property.

(ii) For any compact set $K \subset G$ and any $n$ there exist measurable sets $A_{n}^{K-}, A_{n}^{K+}$ such that

(a) $A_{n}^{K-}+K \subset A_{n}$ and $A_{n}+K \subset A_{n}^{K+}$,

(b) the sequences $\left\{A_{n}^{K-}\right\}$ and $\left\{A_{n}^{K+}\right\}$ have the Følner property,

(c) $\lim _{n \rightarrow \infty}\left(\theta\left(A_{n}^{K-}\right) / \theta\left(A_{n}\right)\right)=\lim _{n \rightarrow \infty}\left(\theta\left(A_{n}^{K+}\right) / \theta\left(A_{n}\right)\right)=1$.

We begin with the following lemma:

Lemma A.3 [1]. Every weakly almost periodic function is amenable. Furthermore, if $\left\{f_{n}\right\}_{n}$ is a sequence of non-negative functions in $L^{1}(G)$ such that

(i) $\int_{G} f_{n}(x) d x=1, \forall n$,

(ii) $\lim _{n}\left\|\delta_{x} * f_{n}-f_{n}\right\|_{1}=0$ for all $x \in G$,

then for any weakly almost periodic function $g$ on $G$ we have

$$
M(g)=\lim _{n} \int_{G} f_{n}(x) g(x) d x .
$$


Proposition A.4. Let $\left\{A_{n}\right\}_{n}$ be a sequence of subsets of $G$ which has the Følner property. Let

$$
f_{n}=\frac{1}{\theta\left(A_{n}\right)} \mathbf{1}_{A_{n}},
$$

where $\mathbf{1}$. is the characteristic function of the set. Then $\left\{f_{n}\right\}$ verifies the conditions from the previous lemma. In particular, for any weakly almost periodic function $g$ on $G$, we have

$$
M(g)=\lim _{n} \frac{\int_{A_{n}} g(x) d x}{\theta\left(A_{n}\right)} .
$$

Proof. It is easy to see that

$$
\left|\delta_{x} * f_{n}-f_{n}\right|=\frac{1}{\theta\left(A_{n}\right)} \mathbf{1}_{A_{n} \Delta\left(x+A_{n}\right)} .
$$

Then the proof follows from the definition of the Følner property.

The next proposition shows that any van Hove sequence has the Følner property. For this we show in fact that

$$
A_{n} \triangle\left(x+A_{n}\right) \subset \partial^{\{x,-x\}}\left(A_{n}\right) .
$$

Proposition A.5. Let $\left\{A_{n}\right\}_{n}$ be a van Hove sequence in G. Then $\left\{A_{n}\right\}_{n}$ has the Følner property.

Proof. Let $x \in G$. Let $K^{\prime}=\{x,-x\}$. We prove that

$$
A_{n} \triangle\left(x+A_{n}\right) \subset \partial^{K^{\prime}}\left(A_{n}\right) .
$$

Let $y \in A_{n} \Delta\left(x+A_{n}\right)$.

First case: $y \in A_{n}, y \notin\left(x+A_{n}\right)$. Since $y \notin\left(x+A_{n}\right)$ then $y-x \notin A_{n}$ so $y-x \in \overline{G \backslash A_{n}}$. Since $x \in K^{\prime}=-K^{\prime}$ we get

$$
y \in-K^{\prime}+\overline{G \backslash A_{n}} .
$$

We know $y \in A_{n}$. Thus $y \in \partial^{K^{\prime}}\left(A_{n}\right)$, hence we are done.

Second case: $y \notin A_{n}, y \in\left(x+A_{n}\right) . \quad y \in\left(x+A_{n}\right)$ implies $y \in K^{\prime}+A_{n}$. Since $y \notin A_{n}$ then $y \notin \operatorname{Int}\left(A_{n}\right)$. Hence $y \in \partial^{K^{\prime}}\left(A_{n}\right)$.

In the next two propositions we show in fact that when we compute the mean by averaging over van Hove sequences, we can ignore what happens in the $K$-boundary of the sequence.

Proposition A.6. Let $\left\{A_{n}\right\}_{n}$ be a van Hove sequence in G. Let $K$ be an arbitrary compact set and let

$$
\begin{aligned}
& B_{n}=A_{n} \backslash\left(\left(-K+\overline{G \backslash A_{n}}\right) \cap A_{n}\right), \\
& C_{n}=(\{0\} \cup K)+A_{n} .
\end{aligned}
$$

Then $B_{n}, C_{n}$ are van Hove sequences. 
Proof. (i) The proof for $B_{n}$. Let $K^{\prime}$ be a compact subset of $G$. Let $K^{\prime \prime}=(\{0\} \cup$ $K)+\left(\{0\} \cup K^{\prime}\right) . K^{\prime \prime}$ is compact. Let $x \in \partial^{K^{\prime}}\left(B_{n}\right)$. Then $x \in K^{\prime}+B_{n} \backslash \operatorname{Int}\left(B_{n}\right)$ or $x \in\left(-K^{\prime}+\overline{G \backslash B_{n}}\right) \cap B_{n}$.

First case: $x \in\left(K^{\prime}+B_{n}\right) \backslash \operatorname{Int}\left(B_{n}\right)$. Then $x \in\left(K^{\prime}+\left(A_{n} \backslash\left(\left(-K+\overline{G \backslash A_{n}}\right) \cap A_{n}\right)\right)\right) \backslash \operatorname{Int}\left(B_{n}\right)$, hence $x \in K^{\prime}+A_{n}$. If $x \notin \operatorname{Int}\left(A_{n}\right)$ we get $x \in \partial^{K^{\prime}}\left(A_{n}\right)$, so $x \in \partial^{K^{\prime \prime}}\left(A_{n}\right)$. If $x \in \operatorname{Int}\left(A_{n}\right)$, since $x \notin \operatorname{Int}\left(B_{n}\right)$ we get $x \notin \operatorname{Int}\left(A_{n} \backslash\left(\left(-K+\overline{G \backslash A_{n}}\right) \cap A_{n}\right)\right)$, so $x \notin \operatorname{Int}\left(A_{n} \cap\left(G \backslash\left(\left(-K+\overline{G \backslash A_{n}}\right) \cap A_{n}\right)\right)\right)$, hence

$$
x \notin \operatorname{Int}\left(A_{n}\right) \cap \operatorname{Int}\left(\left(G \backslash\left(\left(-K+\overline{G \backslash A_{n}}\right) \cap A_{n}\right)\right)\right) .
$$

This implies $x \notin \operatorname{Int}\left(\left(G \backslash\left(\left(-K+\overline{G \backslash A_{n}}\right) \cap A_{n}\right)\right)\right)$, so $x \notin\left(G \backslash \overline{\left.\left(\left(-K+\overline{G \backslash A_{n}}\right) \cap A_{n}\right)\right)}\right.$. Hence

$$
x \in \overline{\left(\left(-K+\overline{G \backslash A_{n}}\right) \cap A_{n}\right)} \subset\left(\left(-K+\overline{G \backslash A_{n}}\right) \cap \overline{A_{n}}\right) \subset \partial^{K}\left(A_{n}\right) .
$$

Thus, we get $x \in \partial^{K}\left(A_{n}\right) \subset \partial^{K^{\prime \prime}}\left(A_{n}\right)$.

Second case: $x \in\left(-K^{\prime}+\overline{G \backslash B_{n}}\right) \cap B_{n}$ implies $x \in B_{n} \Rightarrow x \in A_{n}$. We also know

$$
\begin{aligned}
x & \in\left(-K^{\prime}+\overline{G \backslash B_{n}}\right)=\left(-K^{\prime}+\overline{G \backslash\left(A_{n} \backslash\left(\left(-K+\overline{G \backslash A_{n}}\right) \cap A_{n}\right)\right.}\right) \\
& =-K^{\prime}+\overline{\left(G \backslash A_{n}\right) \cup\left(-K+\overline{\left(G \backslash A_{n}\right)}\right)} \\
& \left.=-K^{\prime}+\overline{\left(G \backslash A_{n}\right)}+(-K \cup\{0\})\right) .
\end{aligned}
$$

Hence $x \in \partial^{K^{\prime \prime}}\left(A_{n}\right)$.

This proves that $B_{n}$ is a van Hove sequence.

(ii) The prooffor $C_{n}$. Let $K^{\prime} \subset G$ be compact. Let $K^{\prime \prime}=(K \cup\{0\})+\left(K^{\prime} \cup\{0\}\right) . K^{\prime \prime}$ is compact. Let $x \in \partial^{K^{\prime}}\left(C_{n}\right)$, then $x \in\left(K^{\prime}+C_{n}\right) \backslash \operatorname{Int}\left(C_{n}\right)$ or $x \in\left(-K^{\prime}+\overline{G \backslash C_{n}}\right) \cap C_{n}$.

First case: $x \in\left(K^{\prime}+C_{n}\right) \backslash \operatorname{Int}\left(C_{n}\right)$. We get $x \in K^{\prime}+C_{n}$ hence $x \in K^{\prime}+(K \cup\{0\})+A_{n} \subset$ $K^{\prime \prime}+A_{n}$. Since $A_{n} \subset C_{n}$ we obtain $\operatorname{Int}\left(A_{n}\right) \subset \operatorname{Int}\left(C_{n}\right)$ so $x \notin \operatorname{Int}\left(A_{n}\right)$. Hence $x \in \partial^{K^{\prime \prime}}\left(A_{n}\right)$.

Second case: $x \in\left(-K^{\prime}+\overline{G \backslash C_{n}}\right) \cap C_{n}$, then $x \in C_{n}$, so $x \in K^{\prime \prime}+A_{n}$. If $x \notin \operatorname{Int}\left(A_{n}\right)$ we are done. If $x \in \operatorname{Int}\left(A_{n}\right)$, we know that $x \in\left(-K^{\prime}+\overline{G \backslash C_{n}}\right)$, so we get $x \in\left(-K^{\prime}+\overline{G \backslash A_{n}}\right)$, hence $x \in \partial^{K^{\prime \prime}}\left(A_{n}\right)$.

Definition A.7. Let $\left\{A_{n}\right\}_{n}$ be a sequence of subsets of $G$. For any compact $K \subset G$ we define

$$
\begin{aligned}
& A_{n}^{K-}=A_{n} \backslash\left(\left(-K+\overline{G \backslash A_{n}}\right) \cap A_{n}\right), \\
& A_{n}^{K+}=A_{n}+(\{0\} \cup K) .
\end{aligned}
$$

Remark A.8. One has $A_{n}^{K-}=A_{n} \backslash \partial^{K}\left(A_{n}\right)$ and $A_{n}^{K+}=A_{n} \cup \partial^{K}\left(A_{n}\right)$.

The previous proposition says that if $\left\{A_{n}\right\}_{n}$ is a van Hove sequence then $\left\{A_{n}^{K+}\right\}_{n}$ and $\left\{A_{n}^{K-}\right\}_{n}$ are van Hove sequences. 
Proposition A.9. Let $\left\{A_{n}\right\}_{n}$ be a van Hove sequence in $G$ and let $K \subset G$ be any compact set. Then

(i) $A_{n}^{K-}+K \subset A_{n}$,

(ii) $\lim _{n \rightarrow \infty}\left(\theta\left(A_{n}^{K-}\right) / \theta\left(A_{n}\right)\right)=\lim _{n \rightarrow \infty}\left(\theta\left(A_{n}^{K+}\right) / \theta\left(A_{n}\right)\right)=1$.

Proof.

(i) Let $x \in A_{n}^{K-}, y \in K$ and $z=x+y$. Suppose that $z \notin A_{n}$ then $z \in G \backslash A_{n}$, so $x=z-y \in\left(-K+\overline{G \backslash A_{n}}\right)$, a contradiction.

(ii) We have $A_{n}^{K-} \subset A_{n} \subset A_{n}^{K-} \cup \partial^{K}\left(A_{n}\right)$, and similarly $A_{n} \subset A_{n}^{K+} \subset A_{n} \cup$ $\partial^{K \cup\{0\}}\left(A_{n}\right)$, with the second inclusion following from the fact that

$$
x \in A_{n}^{K-}, \quad x \notin A_{n} \quad \text { implies } \quad x \notin \operatorname{Int}\left(A_{n}\right), \text { hence } \quad x \in \partial^{K \cup\{0\}}\left(A_{n}\right) .
$$

Now the proof follows from the definition of van Hove sequences.

In Section 5 we need to average over sequences with certain properties. Since it might happen that in some groups there is no van Hove sequence, but there are sequences with these properties, the definition of the $\mathbf{M}$ property is a natural one. In this section we proved that any van Hove sequence has the property $\mathbf{M}$.

\section{References}

1. L. N. Argabright, On the mean of a weakly almost periodic function, Proc. Amer. Math. Soc., 36 (1972), 315-316.

2. L. N. Argabright, J. Gil de Lamadrid, Fourier Analysis of Unbounded Measures on Locally Compact Abelian Groups, Memoirs of the American Mathematical Society, Vol. 145, American Mathematical Society, Providence, RI, 1974.

3. M. Baake, Diffraction of weighted lattice subsets, Canad. Math. Bull., 45 (2002), 483-498.

4. M. Baake, D. Lenz, Dynamical systems on translation bounded measures: pure point dynamical and diffraction spectra, Preprint.

5. M. Baake, R. V. Moody, Weighted Dirac combs with pure point diffraction, J. Reine Angew. Math., 573 (2004), 61-94.

6. C. Berg, G. Forst, Potential Theory on Locally Compact Abelian Groups, Springer-Verlag, Berlin, 1975.

7. E. Bombieri, J. E. Taylor, Quasicrystals, Tilings, and Algebraic number Theory, pp. 241-264, Contemporary Mathematics, Vol. 64, American Mathematical Society, Providence, RI, 1987.

8. D. G. Bourgin, Linear topological spaces, Amer. J. Math., 65 (1943), 637-659.

9. J. M. Cowley, Diffraction Physics, 3rd edn., North-Holland, Amsterdam, 1995.

10. E. F. Eberlein, Weak compactness in Banach spaces I, Proc. Natl. Acad. Sci. USA, 33 (1947), 51-53.

11. E. F. Eberlein, Abstract ergodic theorems and weak almost periodic functions, Trans. Amer. Math. Soc., 67 (1949), 217-224.

12. J. Gil. de Lamadrid, L. N. Argabright, Almost Periodic Measures, Memoirs of the American Mathematical Society, Vol. 85, No. 428, American Mathematical Society, Providence, RI, May 1990.

13. A. Hof, On diffraction by aperiodic structures, Comm. Math. Phys., 169 (1995), 25-43.

14. J. C. Lagarias, Mathematical quasicrystals and the problem of diffraction, in Directions in Mathematical Quasicrystals (M. Baake and R. V. Moody, eds.), pp. 61-93, CRM Monograph Series, Vol. 13, American Mathematical Society, Providence, RI, 2000.

15. Y. Meyer, Algebraic Numbers and Harmonic Analysis, North-Holland, Amsterdam, 1972.

16. R. V. Moody, Meyer sets and their duals, in The Mathematics of Long Range Aperiodic Order (R.V. Moody, ed.), pp. 403-441, NATO ASI Series, Vol. C489, Kluwer, Dordrecht, 1997. 
17. R. V. Moody, Uniform distribution in model sets, Canad. Math. Bull., 45 (2002), 123-130.

18. R. V. Moody, N. Strungaru, Discrete sets and dynamical systems in the autocorrelation topology, Canad. Math. Bull., 47 (2004), 82-99.

19. R.S. Phillips, On weakly compact subsets of a Banach space, Amer. J. Math., 65 (1943), 108-136.

20. W. Rudin, Fourier Analysis on Groups, Interscience, New York, 1962.

21. M. Schlottmann, Cut and project sets in locally compact abelian groups, in Quasicrystals and Discrete Geometry (J. Patera, ed.), pp. 247-264, Fields Institute Monographs 10, American Mathematical Society, Providence, RI, 1998.

22. N. Strungaru, The Bragg spectrum of a Meyer set, Preprint, 2004.

23. A. A. Tempel'man, Ergodic theorems for general dynamic systems, Soviet Math. Dokl., 8 (1967), 12131216.

24. A. A. Tempel'man, Ergodic Theorems for Group Actions: Informational and Thermodynamical Aspects, Mathematics and Its Applications, Vol. 78, Kluwer, Dordrecht, 1992.

Received May 28, 2003, and in revised form March 16, 2004, April 15, 2004, and August 24, 2004.

Online publication January 21, 2005. 